\title{
Finding cells, finding molecules, finding patterns
}

\section{Carolina Wählby*}

Department of Genetics and Pathology,

Centre for Image Analysis,

Uppsala University, Sweden

E-mail: Carolina.Wahlby@cb.uu.se

${ }^{*}$ Corresponding author

\section{Patrick Karlsson}

Centre for Image Analysis, Uppsala University, Sweden

E-mail: Patrick.Karlsson@cb.uu.se

\section{Sara Henriksson, Chatarina Larsson and Mats Nilsson}

Department of Genetics and Pathology, Uppsala University, Sweden E-mail: Sara.Henriksson@genpat.uu.se E-mail: Chatarina.Larsson@genpat.uu.se E-mail: Mats.Nilsson@genpat.uu.se

\section{Ewert Bengtsson}

Centre for Image Analysis,

Uppsala University, Sweden

E-mail: Ewert.Bengtsson@cb.uu.se

\begin{abstract}
Many modern molecular labelling techniques result in bright point signals. Signals from molecules that are detected directly inside a cell can be captured by fluorescence microscopy. Signals representing different types of molecules may be randomly distributed in the cells or show systematic patterns, indicating that the corresponding molecules have specific, non-random localisations and functions in the cell. Assessing this information requires high speed robust image segmentation followed by signal detection, and finally, pattern analysis. We present and discuss these types of methods and show an example of how the distribution of different variants of mitochondrial DNA can be analysed.
\end{abstract}

Keywords: mass data analysis; image analysis; cytometry; single molecule detection; padlock probes; pattern analysis.

Reference to this paper should be made as follows: Wählby, C., Karlsson, P., Henriksson, S., Larsson, C., Nilsson, M. and Bengtsson, E. (2008) 'Finding cells, finding molecules, finding patterns', Int. J. Signal and Imaging Systems Engineering, Vol. 1, No. 1, pp.11-17.

Biographical notes: Carolina Wählby at Uppsala University received her MSc in Molecular Biotechnology 1998 and a PhD in Image Analysis 2003. She is currently an Assistant Professor at the Centre for Image Analysis and part time researcher at the Department of Genetics and Pathology, both at Uppsala University. Her interests include development of algorithms for applied digital image cytometry, and she has 13 international reviewed publications in this field.

Patrick Karlsson is currently working towards his $\mathrm{PhD}$ Degree in Applied Digital Image cytometry at the Centre for Image Analysis. He received his MSc Degree in Engineering Physics from Uppsala University in 2002. He is a member of IAPR.

Sara Henriksson has a Masters Degree in Chemical Engineering and she is currently a $\mathrm{PhD}$ student at the Department of Genetics and Pathology, Uppsala University. Her research interests include design and optimisation of padlock probes for in situ applications. 
Chatarina Larsson is currently a $\mathrm{PhD}$ student at the Department of Genetics and Pathology, Uppsala University. Her research interests include in situ genotyping of single DNA molecules using padlock probes, and she has three international reviewed publications in this field.

Mats Nilsson received his $\mathrm{PhD}$ in Medical Genetics in 1998 and visited Leiden University as a Post Doctoral Fellow in 1999-2000. He is an Associate Professor at the Department of Genetics and Pathology, Uppsala University. His research aims at developing methods for single-cell and single-molecule analysis. The research has resulted in more than 30 peer-reviewed original papers, several book chapters, and four patents. In 2006 he received the Feulgen Prise from the Society for Histochemistry. He is supervisor for seven $\mathrm{PhD}$ students and two post doctoral fellows, and he is on the Board of Directors for Olink AB.

Ewert Bengtsson received his MSc and PhD in Engineering Physics from Uppsala University in 1974 and 1977, respectively. He continued the work on cell image analysis from his thesis, first as a researcher at Uppsala University and then in spin-off companies, from 1983-1988. He returned to Uppsala University as Adjunct Professor and established the centre for image analysis where, since 1995, he is active as a full Professor. His research interests include all kinds of biomedical image analyses. He has published over 120 papers in the field and supervised more than $25 \mathrm{PhD}$ students.

\section{Introduction}

Data mining can be defined as the science of extracting useful information from large data sets. In this case, the input data are digital images of cells captured using fluorescence microscopy, and the information we aim to retrieve is that of spatial distribution patterns of different variants of fluorescence labelled molecular targets. New probing and staining techniques allow a large variety of molecular targets to be visualised in situ and imaged by fluorescence microscopy. Biological processes can be studied at the ultimate level of single molecules, and with sufficient precision to distinguish even closely similar variants of molecules. It is thus possible to study the inter- or sub-cellular context of molecules that otherwise may go undetected at the level of populations of molecules and cells. At the same time, large numbers of cells have to be analysed to retrieve statistically significant information. Extracting information from the resulting image data will, therefore, require efficient and robust cell segmentation as well as signal detection and finally, pattern analysis.

Before signals can be assigned as coming from a particular cell, each cell has to be delineated. Segmentation is the process in which an image is divided into its constituent objects, or parts, and background. Cells can be visualised in many different ways, using different kinds of probes or stains that bind to structures within a cell. It is therefore difficult to define a single algorithm that will always find the individual cells in an image, independent of method for visualisation and cell morphology. Instead, cell image segmentation can be seen as a modelling problem where different approaches more or less explicitly are based on models of the cells. For example, thresholding methods can be seen as being based on a model stating that cells have an intensity that is different from the surroundings. More robust segmentation can be obtained if a combination of features, such as intensity, edge gradients, and cellular shape, is used.
In many applications in cell biology, where fluorescence marked probes are applied, the resulting images are composed of signals, seen as spots of different shapes and intensities. The localisation of these regions can yield important biological information. In multiple labelling experiments in particular, measurements of relative positions of regions labelled with different marker molecules can provide insight into the functional relationship between organelles and/or processes. Visual inspection is, apart from being tedious, beset with various sources of error. The positions of signals in an image should be determined automatically to derive objective information and allow further extraction of image information, such as signal intensity distribution, relative positioning and pattern analysis. The human mind is exceptional at finding patterns; it will even find patterns in data that are completely random. It is, therefore, valuable to have computerised methods that can search for patterns in a more objective way.

We present an image based data mining example where the distribution of different variants of the genetic information contained in mitochondria (i.e., mtDNA) has been examined. MtDNA is present in multiple copies in the mitochondrion of the cell. It is inherited together with the cytoplasm during cell replication. Genetic diseases are often caused by mutations where one single nucleotide has been substituted by another, a so-called point mutation. To be able to study and diagnose such diseases with limited material from patients, there is a need for methods to detect point mutations in situ. Padlock probes and Rolling Circle Amplification (RCA) combine highly specific target sequence recognition with a high signal-to-noise ratio. Padlock probes have been successfully used for detecting point mutations in mitochondrial DNA, by Larsson et al. (2004). We combine cell segmentation, padlock probes, signal detection and pattern analysis to examine the distribution of mtDNAs. These types of methods could also be used in applications ranging from detection of infectious organisms to studies of tumours. 


\section{Methods}

The methods section is divided into three parts, describing methods for segmentation of cells, detection of signals, and evaluation of patterns in the detected signal distribution. A specific example is thereafter brought up in the 'Experiments and results' section.

\subsection{Cell segmentation: finding cells}

The difficulty of the segmentation problem is highly dependent on the type of specimen that is to be analysed, and the result of the segmentation usually determines the eventual success of the final analysis. If we are dealing with cytological specimens where the cells are lying singly on a clean background with well stained nuclei, and if the analysis task is limited to nuclear properties, then a simple automatic thresholding method may be sufficient. Thresholding is often based on histogram characteristics of the pixel intensities of the image, see Sahoo et al. (1988). In order to get a satisfactory segmentation result by thresholding, a sufficiently uniform background is required. The transition between object and background may be diffuse, making an optimal threshold level difficult to find, even after background correction. At the same time, a small change in the threshold level may have a great impact on the further analysis; feature measures such as area and volume are directly dependent on the threshold. Adaptive thresholding, i.e., local automatic thresholding, can be used to circumvent the problem of varying background or as a refinement to a coarse global threshold, see Ortiz de Solorzano et al. (1999). The problems of segmenting clustered objects and choosing a suitable threshold level for objects with unsharp edges will, however, remain.

If we model the objects as consisting of connected regions of similar pixels we obtain region growing methods. A popular region growing method which has proved to be very useful in many areas of image segmentation is the so-called watershed algorithm. The method was originally suggested by Digabel and Lantuéjoul, and extended to a more general framework by Beucher and Lantuéjoul (1979). Watershed segmentation has then been refined and used in very many situations, see Meyer and Beucher (1990) and Vincent (1993) for an overview. If the intensity of the image is interpreted as elevation in a landscape, the watershed algorithm will split the image into regions similar to the drainage regions of this landscape. To avoid over-segmentation, i.e., splitting of the image into too many regions, water can be allowed to rise only from places marked as seeds (Beucher, 1992; Landini and Othman, 2003; Lockett et al., 1998; Meyer and Beucher, 1990; Vincent, 1993). Seeds may be found manually or by automated methods. Over-segmentation can also be reduced by rule-based merging, e.g., shown by Najman and Schmitt (1996).

Cell nuclei are usually convex and fairly round or elliptic and the shape can, therefore, be used as part of the object model. Touching nuclei that are not separated by an intensity threshold can be separated by distance transforming (Borgefors, 1986) the binary image and applying watershed segmentation, see work done by Malpica et al. (1997), Ortiz de Solorzano et al. (1999) and Wählby et al. (2004).

None of the above described methods will alone produce a satisfactory result on the more difficult types of cell and tissue images. We may, for instance, have problems if

- $\quad$ the cells are clustered

- the image background is varying

- there are intensity variations within the cells.

By combining the methods, more powerful models can be created, and more complex segmentation problems solved. Our experience is that the seeded watershed approach is a useful core component in such segmentation models. Complex segmentation methods often require a large number of input parameters that have to be optimised for each type of input data. In case based reasoning, the segmentation step is initiated by classifying each image as belonging to one of a number of pre-defined cases, and input parameters optimised for the particular case are applied during segmentation, see Perner (1999).

\subsection{Signal detection: finding molecules}

The most common method for finding structures such as proteins and organelles in situ is using antibodies labelled with fluorescing molecules. Fluorescence labelled secondary antibodies can be used to amplify the signal and increase signal to noise ratios. The genetic information contained in the DNA in a cell can be stained as a whole using non-specific chemical dyes, or in a more specific way using oligonucleotide probes that search for a particular DNA sequence. Fluorescence In Situ Hybridisation (FISH) is such a method, and can detect larger mutations such as duplications, translocations and deletions, but it is not sensitive enough to distinguish between single nucleotide sequence variations. Primed In Situ Labelling (PRINS) reaction uses a specific primer that will initiate synthesis of DNA from fluorescent labelled nucleotides at the site of sequence detection, see Koch et al. (1988). The method does not, however, give signals from single-copy genes that are distinguishable from noise caused by insertions of fluorescing nucleotides in other places in the genome. In the Oligonucleotide Ligation Assay (OLA), as shown by Landegren et al. (1988), oligonucleotides are hybridised juxtaposed with the junction at the point mutation. If there is a perfect match, the two probes can be enzymatically hybridised and detected. There is, however, a risk of wrong probes being ligated, especially when trying to find many different sequence variants in the same sample. This can be avoided by, instead of using two separate probes, using a single linear probe, a so called padlock probe. The padlock probe has ends that are designed to hybridise juxtaposed at the point mutation and if correctly base paired at the point 
mutation, the two ends can be enzymatically ligated, forming a circular DNA molecule, see Nilsson et al. (1994). The specifically reacted circular DNA can thereafter be amplified using RCA generating molecules that are bound by hundreds of fluorescing probes, see Banér et al. (1998). These signals can be detected by fluorescence microscopy as bright spots at, or below, the resolution of the microscope, the image resolution being limited by the point spread function of the microscope.

An image that contains multiple, and sometimes clustered spots with different maximum intensities, can be segmented in many different ways. Regions found by procedures such as intensity thresholding often contain more than one local maximum of intensity, indicating that the region consists of more than one spot. Top-hat transforms, see Haralick and Shapiro (1992), in combination with threshold procedures fail to divide the image into separate domains, each containing one local maximum of intensity, as the top-hat transform is unable to distinguish a local maximum from a saddle-point. If each spot contains a single local maximum, watershed segmentation, as described above, in combination with a background threshold, may be used to delineate individual signals. Another approach is the largest contour segmentation by Manders et al. (1996), where the domain of each signal is defined by a local maximum and an iterative region-growing. If two or more signals are clustered into a spatially large signal, where the individual signals do not contain individual intensity maxima (due to tight clustering or signal saturation), the shape of the signal can provide clues as to how the signals should be detected. In the work by Karlsson and Lindblad (2004), the curvature of the edge of each signal cluster is examined, and signals are positioned within the cluster starting from the position where the greatest curvature is found.

\subsection{Hypotheses testing: finding patterns}

Patterns in image data can be evaluated by interpreting the signal distribution as image texture, and by using texture measurements. Some of the most commonly used texture measures are derived from the Grey Level Co-occurrence Matrix (GLCM). The GLCM is a tabulation of how often different combinations of pixel brightness values (grey levels) occur in a pixel pair in an image, see Haralick and Shapiro (1992). Multi-interval discretisation has also shown to give useful information for cell pattern classification, see Perner et al. (2002). Different kinds of distance measures can also be used to evaluate spatial relationships between signals once they have been detected. In the case where we want to know whether red and green signals are randomly distributed in the cytoplasm or not, we can simply count how often a red signal has a green signal as its closest neighbour, and how often a red signal has a red signal as its closest neighbour (and the other way around). To evaluate the outcome, we have to know what distributions can be expected. By creating a virtual cell, where possible positions and number of signals of different types are given as inputs, different hypotheses can be tested. We can then compare the spatial relationships between signals in real cells with those in a virtual cell with the same input parameters. Signals in the virtual cell can be positioned based on a hypothesis, i.e., either randomly, or according to a pattern. Thousands of randomised virtual cells can thereafter be created, and the probability of the real cell having the hypothesised signal distribution pattern can be examined. Factors such as staining efficiency and noise may also be added to the virtual cell for comparison.

\section{Experiments and results}

To illustrate the concepts discussed we will, here, describe a project where model based cell segmentation is combined with padlock probing for molecule detection, model based signal detection and pattern analysis, to examine the spatial distribution of mtDNAs.

\subsection{Finding cells}

In the presented experiment, no general stain defining the cytoplasm is available. We do, however, have a general stain defining the nucleus of each cell. Combining this information with the fact that the over all signal variance is higher within the cytoplasm than in the image background, a model defining cytoplasms is created. The three markers (i.e., nuclear stain, padlock probe 1 , and padlock probe 2) are shown as three images, see Figure 1(a)-(c), each captured with a different filter set in the fluorescence microscope. Cells segmentation is initiated by intensity thresholding of the image showing the nuclear stain. A suitable threshold is found using Otsu's method, which searches for the threshold level that minimises the intra-class variance of foreground as well as background, see Otsu (1979). The resulting binary image is shown in Figure 1(d). Intensity thresholding is not enough to separate nuclei that are very close to one another. Thanks to their round shape, touching nuclei can be separated by applying watershed segmentation to a distance map of the binary image. Over-segmentation due to multiple local maxima is avoided by smoothing of the distance map. The distance map is shown in Figure 1(e), and the result after watershed segmentation is shown in Figure 1(f). The region surrounding each nucleus, not belonging to the image background, is the cytoplasm. The image background has less variance than the parts of the image containing cells, and can thus be found by variance filtering. The variance map of the sum of B and C (Figure $1(\mathrm{~g})$ ) is thresholded using Otsu's method. Small, disconnected regions are removed by morphological opening, and larger disconnected regions are re-connected by dilation. In both cases, a disc-shaped structure element of radius 10 pixels was used. The choice of structure element depends on size and density of signals.

Each pixel of the resulting binary image (Figure 1(h)) is assigned to the closest nucleus by seeded watershed segmentation, using the segmentation result from the segmentation of the nuclei as seeds. Non-seeded regions 
are discarded as background. Figure 1(i) shows the final segmentation result on top of a projection of the three input images.

Figure 1 (a) Part of an image of DAPI stained cell nuclei; (b) image of the same cells showing logarithm of signals from padlock probe 1 (stained with $\mathrm{Cy} 3$ ); (c) logarithm of signal from padlock probe 2 (stained with FITC); (d) binary image after thresholding of a; (e) shows the same image after distance transformation; (f) is the result after watershed segmentation on the distance map, i.e., the final segmentation of the nuclei; (g) the cytoplasm is found by combining the two images showing padlock probes (b and c) and applying a variance filter; (h) potential cytoplasm after thresholding of variance map and morphological opening to remove noise and (i) final segmentation result based on shape of nuclei and variance of cytoplasm

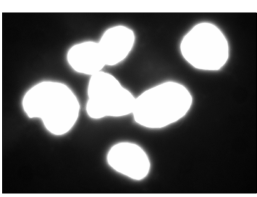

(a)

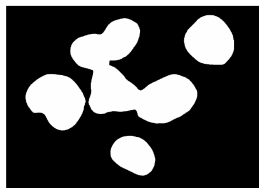

(d)

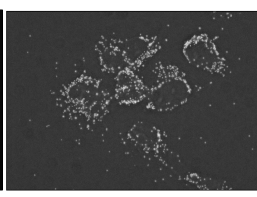

(b)

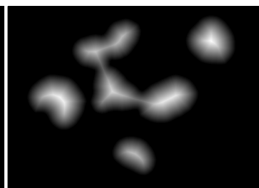

(e)

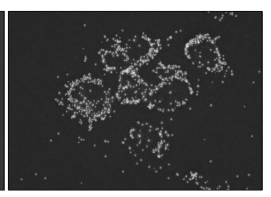

(c)

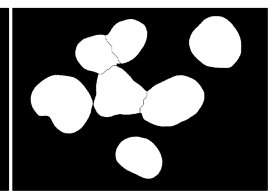

(f)

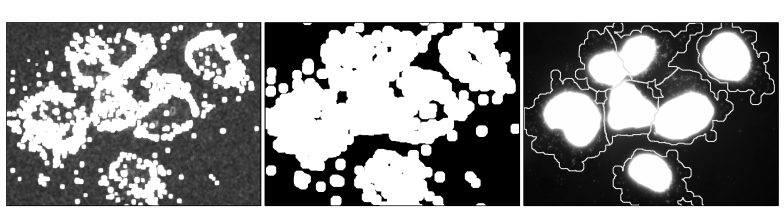

(g)

(i)

\subsection{Finding molecules}

In the presented experiment, a model system with padlock probes was used. It consists of different detection sequences that represent real point mutations. Four different padlock probes were used for testing efficiency of staining and evaluation of signal distribution patterns. Two of the padlock probes hybridise to different sites on the same mtDNA fragment, i.e., they are non-competing. One is detected using Сy3 (red), and one using FITC (green). The other two probes bind to the same site, and are, therefore, competing.

Signal detection was initiated by first reducing the background variation present in the images. As the cells are cultured on a glass surface, they are comparably flat. Despite this, it is necessary to image them in more than one focal plane to make sure that all signals are detected. In the presented study, the slides were studied in a fluorescence microscope (Axioplan II Zeiss) using a 63x objective. Images were collected with Axiovision 4.3 software as a 16 layers $z$-stack with 0.5 um between consecutive layers. The nuclear stain DAPI emission was collected at $360 \mathrm{~nm}$ excitation wavelength for $200 \mathrm{~ms}$, green padlock detection fluorochrome FITC at $470 \mathrm{~nm}$ for $200 \mathrm{~ms}$ and red padlock detection fluorochrome Cy3 at $546 \mathrm{~nm}$ for $450 \mathrm{~ms}$. The background was reduced in each $z$-image separately by morphological tophat filtering, using a disc of radius 10 pixels. Tophat filtering removes intensity variations that have a spatial extent greater than that of the disc. The 16 layers were thereafter combined using maximum intensity projection. Projection of the 3D information to a single image will result in loss of spatial information in the $z$-direction. As the extension of cultured cells in $z$-direction is only a fraction of their extension $i$ $x$-, and $y$ - directions, the 3D information was considered less important. This would, however, not be the case if cells in the tissue were observed.

The result after pre-processing and maximum intensity projection of a small fraction of an image is shown in Figure 2(a). Simple intensity thresholding will separate the signals from the image background, but signals that are clustered will not be separated from each other. To separate clustered signals, Watershed segmentation, starting from all local maxima, is applied to the image, and the watershed regions are allowed to extend until they reach a predefined background threshold. The resulting signal centres after watershed segmentation of Figure 2(a) are shown in Figure 2(b).

Figure 2 (a) Enlarged image showing signals from padlock probes after image pre-processing and (b) positions of detected signals using watershed segmentation

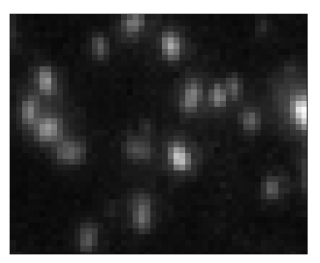

(a)

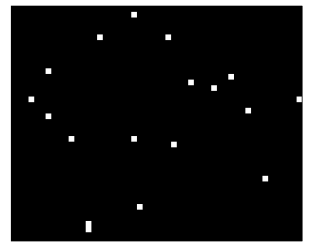

(b)

\subsection{Finding patterns}

The patterns of red and green signal distributions were examined by searching for aggregations of signals, i.e., the existence of groups of signals with the same colour. The affinity of red and green signals was measured as the number of red signals with a green nearest neighbour, the number of red signals with a nearest red neighbour, the number of green signals with a nearest green neighbour, and finally the number of green signals with a nearest red neighbour. To normalise the observed result and evaluate the probability of non-random pattern, virtual cells with truly random patterns were created. Virtual cells with 
random signal distributions were created by keeping the number of red and green signals the same as in the real cell. Red and green signals were then randomised within an area corresponding to the cytoplasm region with the $10 \%$ greatest variance. Virtual cells were re-created 1000 times, neighbourhood relations were examined, and the resulting distributions were compared with observed distributions.

A number of restrictions have to be taken into consideration when creating the virtual cells. First of all there is a limit in closeness between signals in the real data due to the point-spread function. Two signals that are of the same colour will not be separated if they are closer than the width of a single signal. This has to be compensated for in the randomised data, or else it will affect the outcome of the analysis of the neighbour relations. Randomised signals that appeared closer to one another than the two closest signals in the real data were simply removed, and a new pair of random signals was created and tested for closeness with the existing randomised signals. Figure 3(a), top, shows the true signal distribution within the cytoplasm of a cell with competing padlock probes, red and green signals as + and o respectively. Figure 3(a), bottom, shows one of the 1000 virtual cells with randomised signals. Figure 3(b), top, shows the true signal distribution within the cytoplasm of a cell with non-competing padlock probes, red and green signals as + and o respectively. Figure $3(\mathrm{~b})$, bottom, shows one of the 1000 virtual cells with randomised signals in the non-competing case. As can be seen, it is not trivial to pick out the cells showing random distributions compared to those showing a non-random affinity between red and green signals. Comparing the randomised data with the true signal distributions shows that the pattern falls within the randomised distribution in the case with competing probes, while the non-competing probes show a red-green affinity three standard deviations greater than that of the randomised distribution. This agrees with what one would expect as the non-competing probes can bind to the same mtDNA fragment.

Figure 3 Cells treated with two competing: (a) or non-competing and (b) padlock probes in a 50-50 concentration. Top: true signal distribution, bottom: randomised signal distribution. Red and green signals as + and o respectively. Nucleus and cytoplasm outlined

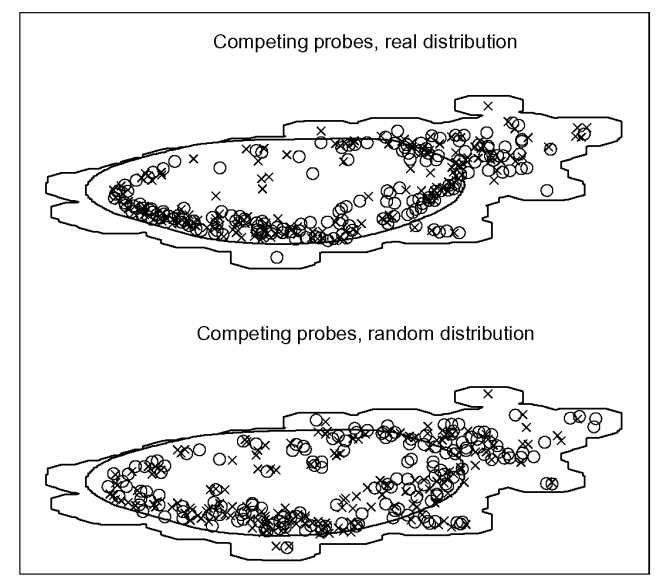

(a)
Figure 3 Cells treated with two competing: (a) or non-competing and (b) padlock probes in a 50-50 concentration. Top: true signal distribution, bottom: randomised signal distribution. Red and green signals as + and o respectively. Nucleus and cytoplasm outlined (continued)

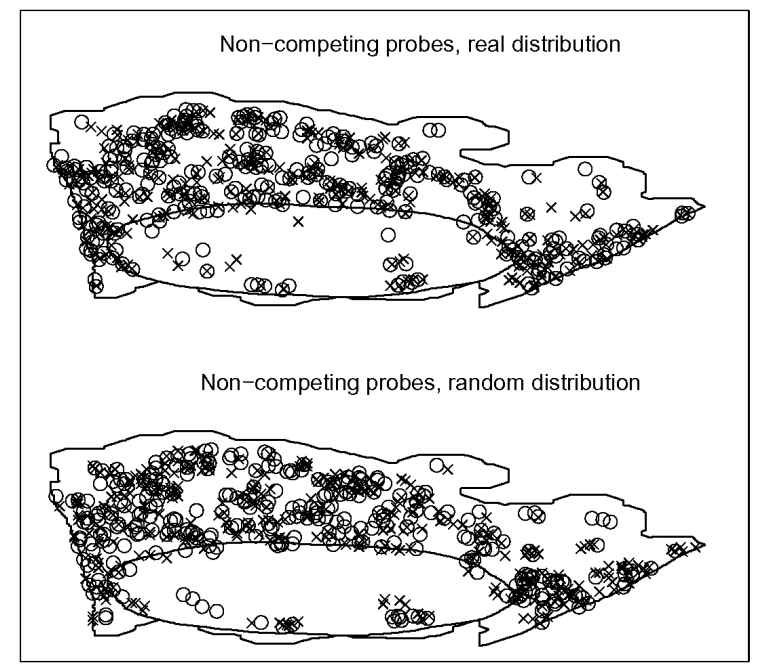

(b)

\section{Conclusions}

Patterns and spatial relationships between molecules in cells are of great interest in many types of analysis. One way of examining patterns in cells is by visualising the molecules of interest using highly specific detection probes, and comparing observed signal distributions with randomised distributions in a virtual cell. Before patterns can be examined, signals from detection probes have to be found, and clustered signals separated. More than one cell is often observed simultaneously, and automated identification of each individual cell in an image provides efficient analysis of large data sets with little impact from observer bias. In order to obtain a successful cell segmentation method it is important to use as much a priori information as possible about the appearance of the objects that are to be segmented, without resorting to models that are too complex or too difficult to train or apply.

\section{References}

Banér, J., Nilsson, M., Mendel-Hartvig, M. and Landegren, U. (1998) 'Signal amplification of padlock probes by rolling circle replication', Nucleic Acid Research, Vol. 26, pp.5073-5078.

Beucher, S. (1992) 'The watershed transformation applied to image segmentation', Scanning Microscopy, Vol. 6, pp.299-314.

Beucher, S. and Lantuéjoul, C. (1979) 'Use of watersheds in contour detection', International Workshop on Image Processing: Real-time and Motion Detection/Estimation, September, Rennes, France.

Borgefors, G. (1986) 'Distance transformations in digital images', Computer Vision, Graphics and Image Processing, Vol. 34, pp.344-371. 
Haralick, R.M. and Shapiro, L.G. (1992) Computer and Robot Vision, Addison-Wesley, Reading, Massachusetts, USA, Vols. 1-2, pp.1291-1294.

Karlsson, P. and Lindblad, J. (2004) 'Segmentation and separation of point like fluorescent markers in digital images', Proceedings of 2004 IEEE International Symposium on Biomedical Imaging, Washington DC, USA, pp.453-470.

Koch, J., Kolvraa, S., Petersen, K., Gregersen, N. and Bolund, L. (1988) 'Oligonucleotide-priming methods for the chromosome-specific labeling of alpha satellite DNA in situ', Chromosoma, Vol. 98, pp.259-265.

Landegren, U., Kaiser, R., Sanders, J. and Hood, L. (1988) 'A ligase-mediated gene detection technique', Science, Vol. 241, pp.1077-1080.

Landini, G. and Othman, I.E. (2003) 'Estimation of tissue layer level by sequential morphological reconstruction', Journal of Microscopy, Vol. 209, No. 2, pp.118-125.

Larsson, C., Koch, J., Nygren, A., Janssen, G., Raap, A.K., Landegren, U. and Nilsson, M. (2004) 'In situ genotyping individual DNA molecules by target- primed rolling-circle amplification of padlock probes', Nature Methods, Vol. 1, pp.227-232.

Lockett, S.J., Sudar, D., Thompson, C.T., Pinkel, D. and Gray, J.W. (1998) 'Efficient, interactive, and three-dimensional segmentation of cell nuclei in thick tissue sections', Cytometry, Vol. 31, pp.275-286.

Malpica, N., Ortiz de Solorzano, C., Vaquero, J.J., Santos, A., Vallcorba, I., Garcia Sagredo, J.M. and del Pozo, F. (1997) 'Applying watershed algorithms to the segmentation of clustered nuclei', Cytometry, Vol. 28, No. 4, pp.289-297.

Manders, E.M.M., Hoebe, R., Strackee, J., Vossepoel, A.M. and Aten, J.A. (1996) 'Largest contour segmentation: a tool for the localization of spots in confocal images', Cytometry, Vol. 23, pp.15-21.

Meyer, F. and Beucher, S. (1990) 'Morphological segmentation', Journal of Visual Communication and Image Representation, Vol. 1, No. 1, pp.21-46.
Najman, L. and Schmitt, M. (1996) 'Geodesic saliency of watershed contours and hierarchial segmentation', IEEE Transactions on Pattern Analysis and Machine Intelligence, Vol. 18, No. 12, pp.1163-1173.

Nilsson, M., Malmgren, H., Samiotaki, M., Kwiatkowski, M., Chowdhary, B.P. and Landegren, U. (1994) 'Padlock probes: circularizing oligonucleotides for localized DNA detection', Science, Vol. 265, pp.2085-2088.

Ortiz de Solorzano, C., Garcia Rodriguez, E., Jones, A., Pinkel, D., Gray, J.W., Sudar, D. and Lockett, S.J. (1999) 'Segmentation of confocal microscope images of cell nuclei in thick tissue sections', Journal of Microscopy, Vol. 193, pp.212-226.

Otsu, N. (1979) 'A threshold selection method from gray-level histograms', IEEE Trans. on System Man and Cybernetics, Vol. 9, No. 1, pp.62-69.

Perner, P. (1999) 'An architecture for a CBR image segmentation system', Journal on Engineering Application in Artificial Intelligence, Engineering Applications of Artificial Intelligence, Vol. 12, No. 6, pp.749-759.

Perner, P., Perner, H. and Müller, B. (2002) 'Mining knowledge for HEp-2 cell image classification', Journal Artificial Intelligence in Medicine, Vol. 26, pp.161-173.

Sahoo, P.K., Soltani, S., Wong, A.K.C. and Chen, Y.C. (1988) 'A survey of thresholding techniques', Computer Vision, Graphics and Image Processing, Vol. 41, pp.233-260.

Vincent, L. (1993) 'Morphological grayscale reconstruction in image analysis: applications and efficient algorithms', IEEE Transactions on Image Processing, Vol. 2, No. 2, pp.176-201.

Wählby, C., Sintorn, I-M., Erlandsson, F., Borgefors, G. and Bengtsson, E. (2004) 'Combining intensity, edge, and shape information for 2D and 3D segmentation of cell nuclei on tissue sections', Journal of Microscopy, Vol. 215, No. 1, pp.67-76. 